\title{
Prevalência de fraturas de platô tibial em pacientes de um hospital público do Piauí
}

\author{
Prevalence of tibial plateau fractures in patients \\ of a public hospital in Piauí
}

\section{Thayze Lima Batista1 ${ }^{1}$ \\ Jáder Luís Coêlho Fernandes Mendes² ${ }^{2}$ Francisco Valmor Macedo Cunha ${ }^{3}$ (D)}

\begin{abstract}
1,2Faculdade UNINASSAU (Teresina). Piauí, Brasil. thayzinhalima00@gmail.com, jadermendes82@hotmail.com
\end{abstract} ${ }^{3}$ Autor para correspondência. Faculdade UNINASSAU (Teresina). Piauí, Brasil. orfeuyeuridice@gmail.com

RESUMO | OBJETIVO: Determinar prevalência de fraturas de platô tibial em pacientes internos em um Hospital Público do Piauí. MATERIAIS E MÉTODOS: Foram analisados 50 prontuários com Fraturas de Platô Tibial de Janeiro de 2017 a Janeiro de 2018. O instrumento de análise foi um questionário composto por informações relevantes, como: sexo, idade, raça, mecanismo de lesão, tipos de fratura e lado acometido, para a coleta dos dados. Para análise dos dados utilizou-se a estatística descritiva com cálculos de médias, desvio padrão e frequências absoluta e relativa. RESULTADOS: A maior incidência das fraturas foi no sexo masculino (72\%). A média de idade dos pacientes foi de $38 \pm 13,86$ anos, vítimas principalmente de acidentes automobilísticos (66\%), sendo o lado mais acometido o direito (70\%) com a incidência maior de fratura oblíqua (48\%). CONCLUSÃO: há maior prevalência de fraturas do platô tibial no sexo masculino, faixa etária de 19-29 anos, vítima de acidente automobilísticos, com fraturas oblíquas.

PALAVRAS-CHAVE: Prevalência. Fraturas. Hospital público. Fraturas de platô tibial.
ABSTRACT | OBJECTIVE: To determine the prevalence of tibial plateau fractures in internal patients at a Public Hospital of Piauí. MATERIALS AND METHODS: Fifty medical records with Tibial Plateau Fractures from January 2017 to January 2018 were analyzed. The analysis instrument was a questionnaire composed of relevant information, such as: sex, age, race, injury mechanism, types of fracture and affected side, for data collection. For data analysis we used descriptive statistics with calculations of means, standard deviation and absolute and relative frequencies. RESULTS: The highest incidence of fractures was in males (72\%). The mean age of the patients was $38 \pm 13.86$ years, mainly victims of automobile accidents (66\%), with the right side most affected (70\%) with the highest incidence of oblique fracture (48\%). CONCLUSION: there is a higher prevalence of fractures of the tibial plateau in males, aged 19-29 years, victim of automobile accidents, with oblique fractures.

KEYWORDS: Prevalence. Fractures. Public hospital. Tibial plateau fractures. 


\section{Introdução}

Apesar do desenvolvimento na área da saúde nos últimos anos ter provocado impacto satisfatório na expectativa de vida da população mundial e brasileira através de avanços na área médica como produção de vacinas, desenvolvimento de medicamentos mais eficazes e de métodos diagnósticos, a partir da década de oitenta, fatores externos passaram a constituir um grave problema de saúde pública ${ }^{1,2,3}$. Segundo a Organização Mundial de Saúde os acidentes de trânsito e quedas podem ser considerados causas externas que afetam a população ${ }^{4}$.

Comumente aos acidentes de trânsito estão associadas as lesões dos ossos as quais ocorrem quando a ação direta ou indireta da força ultrapassa a resistência mecânica do osso resultando em perda da continuidade do osso dividindo-o em dois ou mais fragmentos ${ }^{5}$. Existem casos especiais em que a perda de continuidade do osso não chega ser completa, e a fratura se instala sob a ação de forças relativamente fracas ${ }^{6}$.

Fratura é, portanto, a quebra estrutural na continuidade de um determinado osso, placa epifisária ou uma superfície articular ${ }^{7}$. Dentre todas as fraturas, as de platô tibial correspondem a cerca de 1 a $2 \%$ de todas as fraturas ${ }^{8}$. Tais fraturas resultam de forças compressivas axiais aplicadas externamente combinadas ou não com estresse em valgo ou em varo da articulação do joelho?.

As fraturas de platô tibial constituem risco a integridade funcional do joelho além de possuírem ampla variação para o tempo de consolidação óssea, variando entre 10 semanas e 10 meses, e de possuírem elevada incidência de não consolidação $(34 \%)^{10}$. Además, os custos ao sistema de saúde e previdenciário do país são altos devido às incapacidades momentâneas ou permanentes ocasionadas por tais fraturas ${ }^{11}$. De forma mais precisa, os custos apenas relacionados a acidentes de trânsito somam cerca de 1,0\% do produto interno bruto (PIB) em países em desenvolvimento, como o Brasil ${ }^{12}$.

As fraturas da tíbia, dentre todas as fraturas dos ossos longos, são as mais comuns, com aproximadamente 300.000 casos por ano nos Estados Unidos e 50.000 por ano no Brasil ${ }^{13}$. Embora não existam dados recentes do Ministério da Saúde sobre traumas associados a Fratura de Platô Tibial, levando em consideração a amostragem do estudo de Albuquerque et al. ${ }^{8}$, no qual relata que $52,3 \%$ foram vítimas de acidentes automobilísticos e $22,6 \%$ sofreram lesões associadas, fica explícito a necessidade de ações proativas em busca da redução dos acidentes em trânsito. Além de todos estes aspectos, as fraturas de tíbia repercutem diretamente sobre a qualidade de vida dos pacientes referentes a recuperação funcional e a qualidade da marcha ${ }^{14}$.

A rigidez do joelho é uma das sequelas mais pertinentes nas fraturas de platô tibial, quando não são ressaltados os cuidados iniciais de mobilização articular pós-operatória nos protocolos de reabilitação. A incongruência articular, mau alinhamento axial e instabilidade são limitações, que se não adequadamente corrigidas, resultam em osteoartrose pós-traumática ${ }^{15}$.

Diante do exposto, estudos epidemiológicos se mostram ferramentas essenciais para a abrangência da causa da lesão e desenvolvimento de políticas públicas para mitigar as causas. $O$ objetivo desta pesquisa foi determinar a prevalência de fraturas de platô tibial em um Hospital Público do estado do Piauí bem como identificar fatores como idade, sexo, mecanismo dos traumas, tipos de trauma bem como a etiologia relacionada a fraturas do platô tibial.

\section{Materiais e metódos}

Trata-se de uma pesquisa de caráter documental composta por 50 prontuários relacionados às fraturas de Platô tibial registrados de janeiro de 2017 a janeiro de 2018 de um Hospital Público do Piauí, em Teresina. Tais prontuários foram analisados de forma retrospectiva, descritiva, com abordagem quantitativa dos dados. $\mathrm{O}$ levantamento foi realizado por meio de busca ativa no banco de prontuários do hospital em questão.

A pesquisa ativa na base de prontuários foi realizada setembro a novembro de 2018 por um único pesquisador. A análise dos prontuários foi baseada em uma ficha previamente desenvolvida pelos pesquisadores a fim de obter os seguintes dados: sexo, idade (anos), raça, mecanismo de lesão, tipo de fraturas e lado acometido. 
Os resultados foram tabulados em planilha para posterior produção de gráficos utilizando Software Microsoft Excelß. O levantamento estatístico é de caráter descritivo através da representação por frequências absoluta e relativa.

O estudo atendeu às normas para a realização de pesquisa em seres humanos através da resolução $N^{\circ} 466 / 12$, tendo sido previamente aprovada pelo Comitê de Ética em Pesquisa (CEP) do Centro Universitário Maurício de NassauUNINASSAU, conforme Parecer № 2805162, CAE 95097318.2.0000.5193 (ANEXO 1) e do Hospital Público do Piauí.

\section{Resultados}

Nesse estudo foram analisados 50 prontuários de pacientes que passaram por um Hospital Público do Piauí após sofrerem algum trauma resultando em fratura de platô tibial. As informações colhidas estão representadas em gráficos e tabelas, onde os resultados foram apresentados em dados quantitativos e percentuais.

A idade média da amostra foi de 38 × 13,86. A distribuição por faixa etária está representada na tabela 1.

Tabela 1. Frequência absoluta e relativa do sexo, idade e mecanismo de lesão de fraturas do platô tibial segundo prontuários de um hospital público do estado do Piauí

\begin{tabular}{llll}
\hline Variáveis Analisadas & & Frequencia absoluta $(\mathrm{n})$ & Frequência relatira (\%) \\
\hline \multirow{2}{*}{ Sexo } & Masculino & 36 & $72 \%$ \\
& Feminino & 14 & $28 \%$ \\
\hline \multirow{4}{*}{ Mecanismo de lesão } & Acidente automobilístico & 33 & $66 \%$ \\
& Queda da própria altura & 07 & $14 \%$ \\
& Queda na prática & 06 & $12 \%$ \\
& desportiva & 04 & $08 \%$ \\
\hline \multirow{3}{*}{ Idade } & Outros & 29 & $58 \%$ \\
& $19-29$ anos & 8 & $16 \%$ \\
\hline
\end{tabular}

De acordo com a tabela 1, observa-se que dos 50 prontuários analisados, 34 são de pacientes masculinos (72\%). Ainda de acordo com a tabela 1, é possível observar que a maior prevalência no mecanismo de lesão das Fraturas de Platô Tibial é de acidentes automobilísticos $33(66 \%)$, seguida de queda da própria altura 7 (14\%), queda na prática desportiva $6(12 \%)$ e outros $4(8 \%)$.

O gráfico 1 representa a classificação por tipo de fratura observadas nos prontuários analisados. Desta forma, 24 era do tipo oblíqua (48\%), seguido por 19 transversa (38\%), 4 espiral (8\%), 1 cominutiva (2\%), 1 fechada (2\%) e 1 exposta (2\%). Sendo o lado acometido mais evidente o direito com 35 (70\%). 


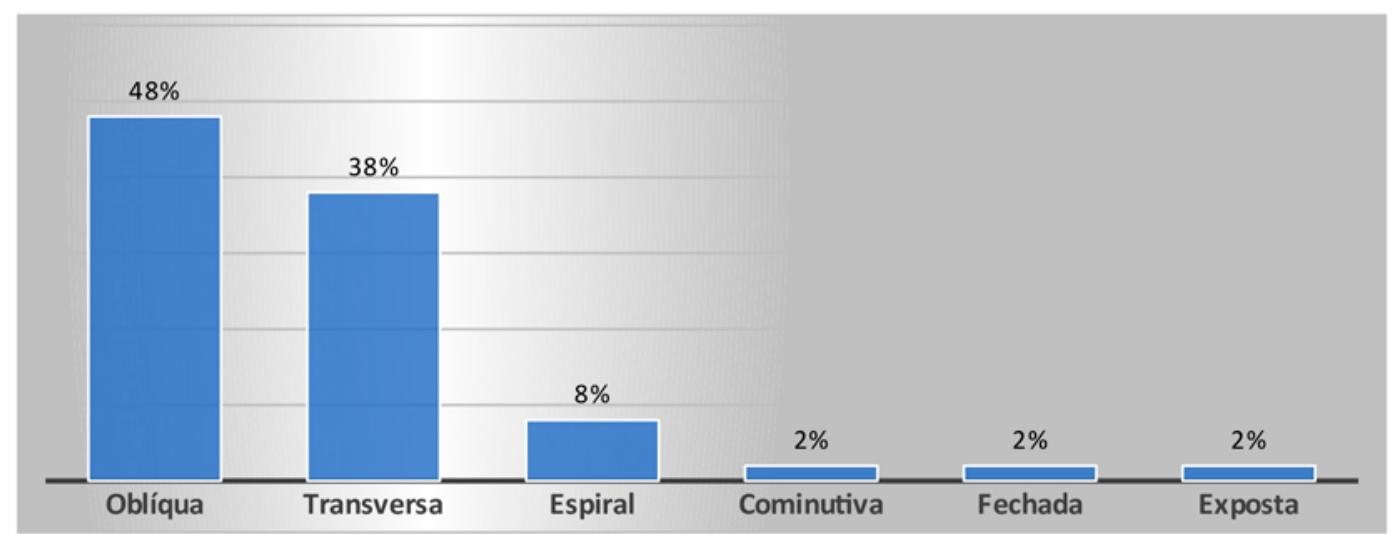

\section{Discussão}

De acordo com Albuquerque et al. ${ }^{8}$, o predomínio do sexo masculino se da pelo fato de suas maiores vulnerabilidades e exposição a situações de risco, o emprego de maior força física durante as atividades físicas e trabalhos braçais ou o perigoso desejo à velocidade e ao desrespeito às leis de trânsito.

Dados semelhantes aos encontrados neste estudo foram relatados por Júnior et al. ${ }^{16}$ que evidenciou prevalência no sexo masculino de $85,2 \%$ de fraturas diafisárias de tíbia em 123 pacientes vítimas de acidentes automobilísticos, justificando a prevalência masculina, pelo fato de se submeterem a situações arriscadas, consumo de bebidas alcoólicas, entre outros. De forma semelhante, neste estudo, a maior causa de fraturas de platô tibial também ocorreu por acidentes automobilísticos.

Em estudo também realizado no estado do Piaui, avaliando o perfil de vítimas de acidentes motociclísticos notou-se que a faixa etária com maior prevalência foram a de 15 a 24 anos e de 25 a $34^{17}$. Tal resultado apresentado vai de encontro aos resultados encontrados neste estudo em que a faixa etária com maior prevalência foi a de 19 a 29 anos (Tabela 1).

Ainda sobre a faixa etária, pacientes idosos que apresentam fraturas causadas por traumas de menor energia (queda da própria altura), de acordo com a classificação AO (método alfanumérico da classificação das Fraturas de Platô Tibial) possivelmente pode estar relacionado a diminuição da densidade mineral óssea ${ }^{16}$.

De acordo com Watson et al. ${ }^{18}$, nos pacientes mais jovens, os fragmentos tendem a ser maiores e em "cunha", devido às forças de cisalhamento (cargas tangenciais e opostas aplicadas em uma direção angular), e nos pacientes idosos é mais comum encontrarmos fraturas por depressão pura, sendo geralmente causadas por traumas de menor energia.

Como visto na tabela 1, os principais mecanismos lesionais das fraturas de platô tibial encontradas neste estudo foram acidentes automobilísticos 33 (66\%), seguida de queda da própria altura 7 (14\%), queda na prática desportiva $6(12 \%)$ e outros $4(8 \%)$.

Neste sentido, Marín-Leon et al. ${ }^{19}$ constataram aumento do número de acidentes automobilísticos em Campinas, onde a frota de automóveis aumentou de 39 para 61 para cada 100 habitantes com especial contribuição das motocicletas por serem mais rápidas no trânsito, pelo baixo custo e a facilidade do financiamento desses veículos.

A rápida urbanização, fadiga dos motoristas, desobediência das sinalizações, excesso de velocidade, ingestão de bebidas alcoólicas e outras drogas nos países em desenvolvimento são as causas que contribuem para o aumento dos acidentes de trânsito, o que torna necessário o desenvolvimento de políticas públicas a fim de mitigar tais fatores ${ }^{20}$.

Quanto ao tipo de fratura, o mais comum encontrado neste estudo foi a com traçado oblíquo (Figura 1). A fratura oblíqua decorre quando uma extremidade óssea é submetida a uma torção ou giro súbito, no mesmo momento em que a outra extremidade permanece fixa, por esse motivo ele se torna em maior incidência nos acidentes automobilísticos, devido ao trauma ser de maior energia por desestabilizar as articulações de joelho ${ }^{21}$. 
Dentre as fraturas de platô tibial, as mais observadas são de moderada a alta energia: as lesões bicondilares e a combinação de cisalhamento e depressão pura de platô tibial lateral e geralmente associam-se a lesões meniscais e/ou ligamentares ${ }^{18}$.

É importante salientar que trata-se de um estudo com amostra reduzida (50 prontuários) e recorte temporal relativamente curto ( 1 ano) além de abranger apenas um hospital que recebe traumas ortopédicos no estado do Piaui. Como viés pode-se citar que o Hospital do estudo é referência no estado para traumas não graves o que pode ter direcionado os resultados aqui apresentados para maior prevalência de traumas advindos de acidentes automobilísticos.

Desta forma, há a necessidade de explorar mais sobre o tema, com mais pesquisas científicas para poder distinguir os fatores de risco e criar metas e ações sociais visando a necessidade de promoção de informações de utilidade pública, medidas preventivas, campanhas de fiscalização e de educação de trânsito, para evitar mais acidentes e conscientizar sobre a necessidade da segurança no trânsito visando reduzir o número de lesões, melhorar o atendimento ao paciente vítima de fratura do platô tibial e diminuir o número de acidentados e diminuir o tempo de afastamento das atividades laborais e sociais bem como diminuir as complicações.

\section{Conclusão}

Na população estudada, foi observado que há maior prevalência de fraturas do platô tibial no sexo masculino, faixa etária de 19-29 anos, vítima de acidente automobilísticos, com fraturas oblíquas, tendo o lado acometido mais presente o direito.

Ressalta-se a necessidade de novas pesquisas clínicas a fim de elucidar o perfil epidemiológico do paciente com fratura de platô tibial além de aumentar o corpo teórico acerca do assunto ainda pouco explorado. Enfatiza-se ainda a relevância de novas pesquisas que abordem a evolução funcional, as possíveis intercorrências e impactos sobre a qualidade de vida de tais pacientes a fim de fomentar políticas públicas para prevenção e protocolos de tratamento para acelerar o retorno as atividades e melhora da qualidade de vida.

\section{Contribuições dos autores}

Cunha FVM participou da concepção, análise estatística, discussão e correção. Batista TL e Mendes JLCF participaram da concepção, coleta e redação do manuscrito.

\section{Conflitos de interesses}

Nenhum conflito financeiro, legal ou político envolvendo terceiros (governo, empresas e fundações privadas, etc.) foi declarado para nenhum aspecto do trabalho submetido (incluindo, mas não se limitando a subvenções e financiamentos, participação em conselho consultivo, desenho de estudo, preparação de manuscrito, análise estatística, etc.).

\section{Referências}

1. Mendes ACG, Sá DA, Miranda GMD, Lyra TM, Tavares RAW. Assistência pública de saúde no contexto da transição demográfica brasileira: exigências atuais e futuras. Cad Saúde Pública. 2012;28(5):955-964. doi: 10.1590/S0102311X2012000500014

2. Campolina AG, Adami F, Santos JLF, Lebrão ML. A transição de saúde e as mudanças na expectativa de vida saudável da população idosa: possíveis impactos da prevenção de doenças crônicas. Cad Saúde Pública. 2013;29(6):1217-1229. doi: 10.1590/ S0102-311X2013000600018

3. Camargos MCS, Gonzaga MR. Viver mais e melhor? Estimativas de expectativa de vida saudável para a população brasileira. Cad Saúde Pública. 2015;31(7):1460-1472. doi: 10.1590/0102311X00128914

4. Lemos CAG, Jorge MT, Ribeiro LA. Perfil de vítimas e tratamento de lesões por causas externas segundo atendimento pelo Centro de Reabilitação Municipal de Uberlândia, MG - Causas externas e fisioterapia. Rev Bras Epidemiol. 2013;16(2): 482-92. doi: $10.1590 /$ S1415-790X2013000200022

5. Loi F, Córdova LA, Pajarinen J, Lin TH, Yao Z, Goodman SB. Inflammation, fracture and bone repair. Bone. 2016;86:119-30. doi: $10.1016 /$ j.bone.2016.02.020

6. Dutton M. Fisioterapia ortopédica, exame, avaliação e intervenção. 2.ed. Porto Alegre: Artmed; 2010.

7. Kisner C, Colby LA. Exercícios Terapêuticos. 5.ed. Barueri: Manole; 2009.

8. Albuquerque RP, Hara R, Prado J, Schiavo L, Giordano V, Amaral NP. Estudo epidemiológico das fraturas do platô tibial em Hospital de Trauma nível I. Acta Ortop Bras. 2013; 21(2):109-15. doi: 10.1590/S1413-78522013000200008 
9. Kfuri Júnior M, Fogagnolo F, Bitar RC, Freitas RL, Salim R, Paccola CAJ. Fratura do Planalto Tibial. Revista Brasileira de Ortopedia. 2009;44(6):468-474. doi: 10.1590/S0102-36162009000600002

10. Joslin CC, Eastaugh-Waring SJ, Hardy JR, Cunningham JL. Weight bearing after tibial fracture as a guide to healing. Clin Biomech. 2008; 23(3):329-333. doi: 10.1016/j. clinbiomech.2007.09.013

11. Fonseca MA. Impacto das fraturas de membros inferiores na qualidade de vida de indivíduos adultos [tese]. Salvador: Escola Bahiana de Medicina e Saúde Pública; 2016.

12. Itami LT, Faro ACM, Meneghin P, Leite RCBO, Silveira CT. Adultos com fraturas: das implicações funcionais e cirúrgicas à educação em saúde. Rev Esc Enferm USP. 43(Esp 2):1238-43. doi: 10.1590/S0080-62342009000600016

13. Sunada EE, Ejnisman L, Leal RD, Pailo AF, Malavolta EA, Sakaki $\mathrm{MH}$ et al. Estudo biomecânico da rigidez da osteossíntese com placas em ponte em tíbias de cadáveres humanos. Acta Ortop Bras. 2010;18(2):66-70. doi: 10.1590/S1413-78522010000200001

14. Nascimento OR, Cemin FS, Morais M, Barroco RS, Fujiki EW, Milani C. Avaliação da qualidade de vida em pacientes com fratura da tíbia. Acta Ortop Bras. 2009;17(4): 211-214. doi: 10.1590/S1413$\underline{78522009000400003}$

15. Stevens DG, Beharry R, Mckee MD, Wadell JP, Schemitsch $\mathrm{EH}$. The long-term funciona lout come of operatively treated tibial plateau fractures. J Orthop Trauma. 2001;15(5):312-20. doi: 10.1097/00005131-200106000-00002

16. Vieira Júnior ST, Aguiar Júnior AP, Sombra LP, Castro JOA, Alves FRV. Epidemiologia das Fraturas diafisárias de tíbia em um hospital municipal de referência em traumatologia. Rev Med UFC. 2017;57(3):12-17. doi: 10.20513/2447-6595.2017v57n3p12-17

17. Santos AMR, Moura MEB, Nunes BMVT, Leal CFS, Teles JBM. Perfil das vítimas de trauma por acidente de moto atendidas em um serviço público de emergência. Cad Saúde Pública. 2008; 24(8):1927-38. doi: 10.1590/S0102-311X2008000800021

18. Watson JT, Schatzker J. Tibial plateau fractures. In: Browner BD, Jupiter JB, Levine AM, Trafton PG, editors. Skeletal trauma. Basic science, management, construction. Philadelphia: Saunders; 2003.

19. Marín-León L, Belon AP, Barros MBA, Almeida SDM, Restitutti MC. Tendência dos acidentes de trânsito em Campinas, São Paulo, Brasil: importância crescente dos motociclistas. Cad Saúde Pública. 2012; 28(1):39-51. doi: 10.1590/S0102311X2012000100005

20. Ministério da Saúde. Redução da morbimortalidade por acidentes de trânsito: mobilizando a sociedade e promovendo a saúde. [Internet]. 2001. Disponível em: http://bvsms.saude.gov.br/ bvs/publicacoes/prog_reducao_acidentes.pdf
21. Prentice WE. Fisioterapia na prática esportiva uma abordagem baseada em competências. 14.ed. Porto Alegre: AMGW; 2012. 